
April 1938

\title{
PHOTOCHEMICAL REACTIONS OF WOOL
}

\author{
By Milton Harris and Arthur L. Smith ${ }^{1}$
}

\begin{abstract}
In an earlier paper, the authors suggested that in the photochemical deterioration of wool, a portion of the sulfur is converted to hydrogen sulfide, some of which is subsequently oxidized to sulfuric acid. The present paper describes an apparatus which was developed for the purpose of segregating these reactions and testing the hypothesis. The results obtained with it show that in an atmosphere of purified nitrogen, hydrogen sulfide is evolved from wool during irradiation. In the presence of air the bulk of the hydrogen sulfide is oxidized to sulfuric acid. The rate of evolution of hydrogen sulfide is increased by the presence of moisture. The data suggest that the primary action of the radiation is an activation of the disulfide group, resulting in a change in the state of the sulfur. A secondary process, involving the evolution of hydrogen sulfide from the irradiated wool then occurs. Water does not appear to be essential to the process which involves changing the state of the sulfur, but it may play an important part in the secondary reactions.
\end{abstract}

CONTENTS

I. Introduction

II. Description of apparatus and technique of operation

III. Experimental results

IV. Discussion_._.

V. References

\section{INTRODUCTION}

In an earlier paper [1] ${ }^{2}$, on the effect of light on wool, the authors suggested that the result of irradiation is the conversion of a portion of the sulfur to hydrogen sulfide, some of which is subsequently oxidized to sulfuric acid. In order to test this hypothesis, it was necessary to devise experiments which would segregate those reactions. The present paper describes an apparatus which was developed for this purpose. The results obtained with it are used to further elucidate the nature of some of the photochemical reactions in wool.

1 Research Associate at the National Bureau of Standards, representing the American Association of Textile Chemists and Colorists. This work was aided by grants from the Textile Foundation, Inc., and the Evanson Levering Co. The present address of Arthur L. Smith is the Evanson Levering Co., Camden, N. J.

Numbers in brackets indicate literature references at the end of the paper. 


\section{DESCRIPTION OF APPARATUS AND TECHNIQUE OF OPERATION}

A schematic diagram of the apparatus for exposing the wool under controlled atmospheric conditions, to the radiation of a glass-enclosed carbon $\operatorname{arc}^{3}$ is shown in figure 1. Gases which may be evolved from the fibers are removed by carrier gases and absorbed by suitable reagents. During an exposure of a sample, the carrier gas is introduced at $A$ and passes over the mercury safety valve $B$, through the trap $C$, and then through the absorption towers $D$ and $E$. The latter have a capacity of about $800 \mathrm{ml}$ each and are equipped with platinum disks for distributing gas similar to those described by Shepherd [2] for gas-absorption pipettes. The disks are perforated plates, each $0.05 \mathrm{~mm}$ thick and $20 \mathrm{~mm}$ in diameter, and with 212

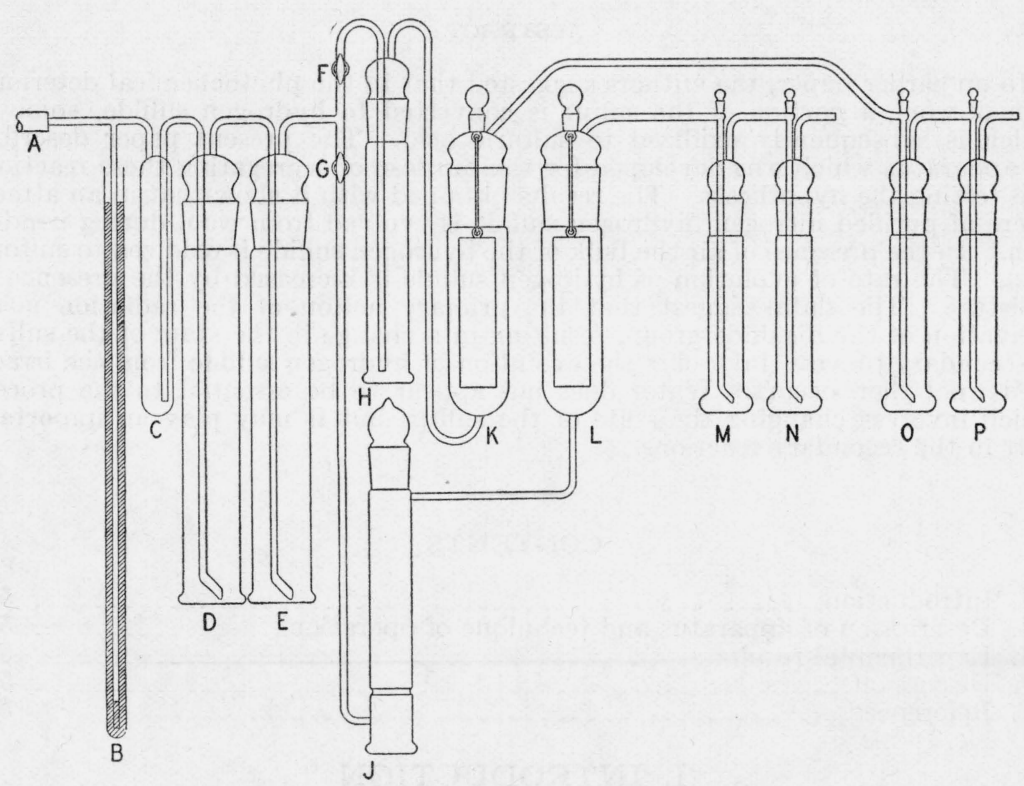

Figure 1.-Schematic diagram of the apparatus for exposing wool under controlled atmospheric conditions to the radiation of a glass-enclosed carbon arc.

evenly spaced holes, $0.06 \mathrm{~mm}$ in diameter. When nitrogen is used as the carrier gas, $D$ and $E$ are filled with an alkaline solution of pyrogallol. These towers do not need to be filled when air is used. However, it was found that the presence of liquid in them facilitates the regulation of the flow of the gas. Furthermore, if a saturated solution of potassium hydroxide is used in the second tower $E$, the air is partially dried, thus permitting the material in the drying tower $J$ to be used for a longer period of time. After the gas leaves $E$, it is divided into two portions, the relative amounts of each being regulated by stopcocks $F$ and $G$. Part of the gas passes through $H$, which is filled with distilled water, and the remainder is dried by passing through $J$, which is filled with anhydrous magnesium per-

3 Fade-Ometer, Atlas Electric Devices Co., Chicago, Ill. 
chlorate. ${ }^{4}$ From them the gas passes through the exposure tubes $K$ and $L$, which contain the wool samples.

The gas emerging from each of the tubes $K$ and $L$, finally passes through two small absorption towers $M N$ and $O P$, in series. These towers have a capacity of $250 \mathrm{ml}$ each and are constructed the same as the larger ones, $D$ and $E$. In addition, they are fitted with groundglass stoppers on the intake tubes to facilitate washing of the inside of the platinum distributors. Since each gas was determined in a

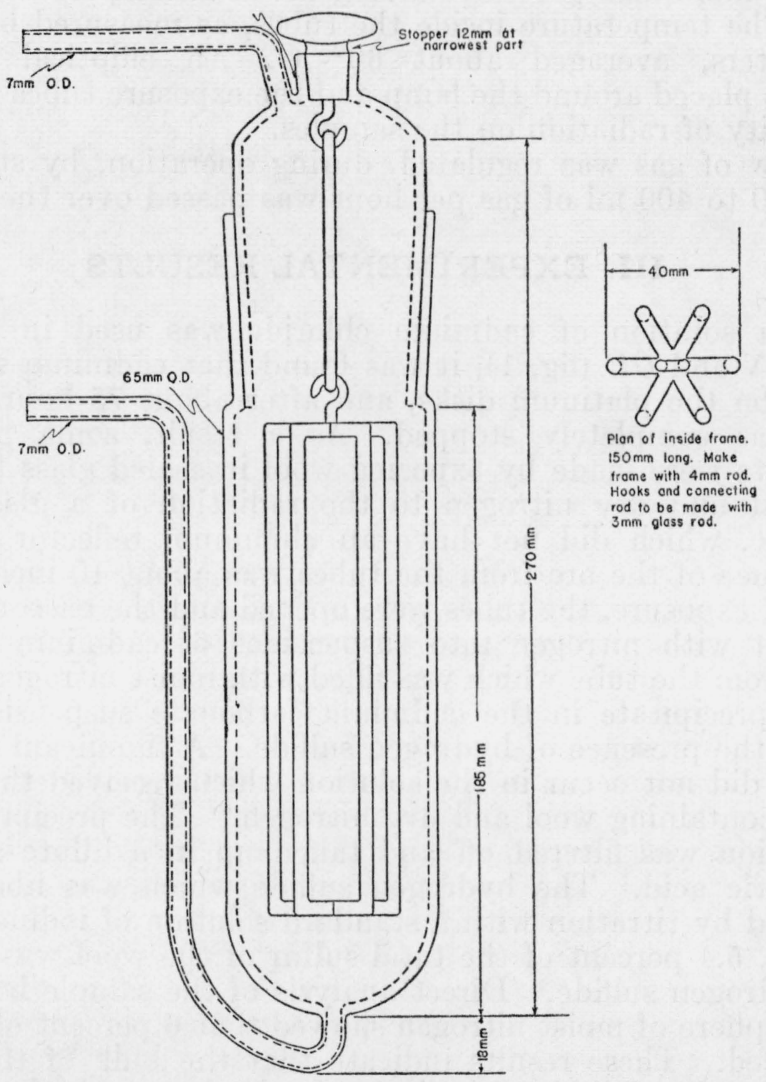

FIGURE 2.-Detailed drawing of the hexagonal glass framework on which the wool is mounted, and of the tube in which the wool is exposed.

separate experiment the towers $M N$ and $O P$ were filled with the same solution. The solutions were as follows: $0.1 \mathrm{~N}$ solution of hydrochloric acid for the absorption of ammonia; a solution containing $10 \mathrm{~g}$ of sodium hydroxide and $10 \mathrm{~g}$ of bromine per liter for tbe absorption of hydrogen sulfide; and a 15-percent solution of cadmium chloride for determining whether the evolved gas was hydrogen sulfide or sulfur dioxide.

Figure 2 is a detailed drawing of the exposure tubes $K$ and $L$.

4 It was shown that when 210 liters of air, saturated with moisture, was dried by passing over anhydrous magnesium perchlorate at $25^{\circ} \mathrm{C}$ at the rate of from 1.5 to 3.5 liters per hour, the residual air did not contain a weighable amount of water [9]. 
Single-ply wool yarn ${ }^{5}$ of very low twist was wound on the bexagonal glass framework. The ground-glass stopper, from which the frame is suspended, permits the frame to be revolved at frequent intervals, which assures fairly uniform exposures. Difficulties encountered in using ordinary lubricants in the large ground-glass joint, were overcome by using a glycol-phthalic anhydride resin [3].

The exposure tubes are 10 inches from the carbon arc. A fan, regulated by a bimetallic thermostat, is mounted below the exposure tubes and maintains the surrounding air at a temperature of about $35^{\circ} \mathrm{C}$. The temperature inside the tubes, as measured by mercury thermometers, averaged about $60^{\circ}$ C. An elliptical aluminum reflector is placed around the lamp and the exposure tubes to increase the intensity of radiation on the samples.

The flow of gas was regulated, during operation, by stopcocks $F$ and $G ; 300$ to $400 \mathrm{ml}$ of gas per hour was passed over the wool.

\section{EXPERIMENTAL RESULTS}

When a solution of cadmium chloride was used in absorption towers $M N$ and $O P$ (fig. 1), it was found that cadmium sulfide precipitated on the platinum disks, and after about 75 hours, the flow of gas was completely stopped. As a result, some preliminary experiments were made by exposing wool in sealed glass tubes filled with moist and dry nitrogen to the radiation of a glass-enclosed carbon arc, which did not have an aluminum reflector around it. The distance of the arc from the tubes was about 10 inches. After 575 hours' exposure, the tubes were opened and the gaseous contents driven out with nitrogen into suspensions of cadmium carbonate The gas from the tube which was filled with moist nitrogen produced a yellow precipitate in the cadmium carbonate suspensions, which indicated the presence of hydrogen sulfide. A significant amount of yellowing did not occur in the solution which received the gas from the tube containing wool and dry nitrogen. ${ }^{6}$ The precipitate in the first solution was filtered off and taken up in a dilute solution of hydrochloric acid. The hydrogen sulfide which was liberated was determined by titration with a standard solution of iodine. By this procedure, 5.4 percent of the total sulfur of the wool was accounted for as hydrogen sulfide. Direct analysis of the sample irradiated in the atmosphere of moist nitrogen showed that 6 percent of the sulfur was evolved. These results indicate that the bulk of the sulfur is evolved from wool during irradiation in the form of hydrogen sulfide and not as sulfur dioxide, ${ }^{7}$ as suggested by King [4] and by Meunier and Rey [5].

The rate at which hydrogen sulfide was evolved during irradiation in nitrogen was determined. The wool was wound on the hexagonal glass frames and dried at $105^{\circ} \mathrm{C}$ for 6 hours, after which it was rapidly transferred to the tubes $K$ and $L$. The latter were covered with black paper, the light was turned on, and dry nitrogen passed over one sample and moist nitrogen over the other for 18 hours. The

\footnotetext{
The yarn was prepared from raw wool which had been extracted with Stoddard solvent and washed with water. It was further purified by extraction in a Soxhlet apparatus with alcohol and ether, for 6 hours with each, and finally washed with water at $50^{\circ} \mathrm{C}$.

w A comparison of the amount of sulfur lost from the sample irradiated in an atmosphere of moist nitrogen under these conditions with that lost by a sample under similar conditions irradiated in the new apparatus described in this paper indicates that the intensity of the radiation is greatly increased by the aluminum reflector.

7 If any sulfur dioxide were present, it would react with hydrogen sulfide as follows: 
paper was then removed and the exposures were started. The absorption pipettes $M N$ and $O P$ contained the alkaline bromine solution and any sulfide sulfur evolved was immediately oxidized to sulfate. At various intervals these solutions were removed and the amount of sulfate was determined as barium sulfate. The rates at which sulfur, in the form of hydrogen sulfide, is evolved from the wool are shown in figure 3.

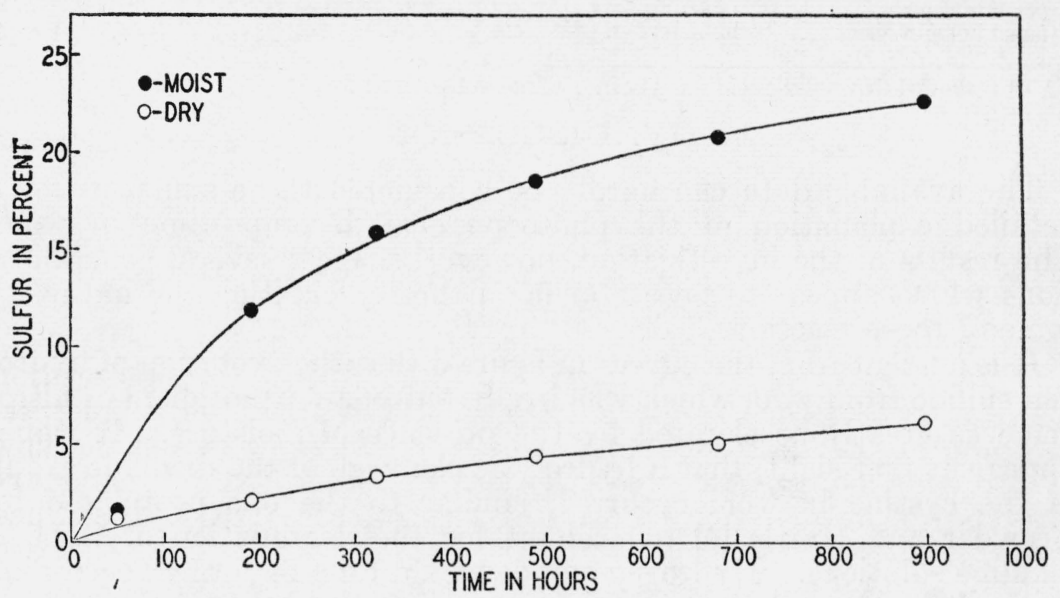

FIGURE 3.-Rate of evolution of hydrogen sulfide from samples of wool exposed in atmospheres of moist and dry nitrogen to the radiation of a glass-enclosed carbon arc.

The ordinate values are in terms of the percentage of the total sulfur which was evolved as hydrogen sulfide during the irradiation.

After 900 hours' exposure, the wool yarn was removed, cut into lengths about one-eighth inch long, and thoroughly mixed to assure uniform sampling. Analyses made on the different samples are shown in table 1.

A similar exposure, using a $0.1 \mathrm{~N}$ solution of hydrochloric acid in tubes $M N$ and $O P$ showed that in 305 hours' exposure, no appreciable amount of ammonia was evolved from either sample.

A third series of exposures of wool in atmospheres of moist and dry air were made. Under these conditions, relatively little hydrogen sulfide was found. The effect of 266 hours' exposure is shown in table 2 .

TABLE 1.-Effect on wool of 900 hours' exposure in atmospheres of moist and dry nitrogen to the radiation from a glass-enclosed carbon arc

\begin{tabular}{|c|c|c|c|c|c|c|c|c|}
\hline Sample & $\begin{array}{c}\text { Total } \\
\text { nitrogen }\end{array}$ & $\begin{array}{l}\text { Ammo- } \\
\text { nia ni- } \\
\text { trogen }\end{array}$ & Cystine & $\begin{array}{l}\text { Total } \\
\text { sulfur }\end{array}$ & $\begin{array}{c}\text { Sulfur } \\
\text { found in } \\
\text { gases }\end{array}$ & $\begin{array}{c}\text { Total } \\
\text { sulfur ac- } \\
\text { counted } \\
\text { for }\end{array}$ & $\begin{array}{l}\text { Alkali } \\
\text { solubil- } \\
\text { ity }\end{array}$ & $\begin{array}{l}\text { Sulfur as } \\
\text { sulfuric } \\
\text { acid }\end{array}$ \\
\hline $\begin{array}{l}\text { Unexposed } \\
\text { Exposed in dry nitrogen } \\
\text { Exposed in moist nitrogen.-. }\end{array}$ & $\begin{array}{l}\% \\
16.22 \\
16.17 \\
16.26\end{array}$ & $\begin{array}{r}\mathrm{mg} / \mathrm{g} \\
0.5 \\
.7 \\
.6\end{array}$ & \begin{tabular}{l}
\multicolumn{1}{l}{$\%$} \\
10.7 \\
4.2 \\
2.9
\end{tabular} & \begin{tabular}{l|l}
$\%$ \\
3.41 \\
3.17 \\
2.42
\end{tabular} & $\begin{array}{c}\% \\
0.22 \\
.79\end{array}$ & $\begin{array}{c}\% \\
100.0 \\
97.2 \\
192.0\end{array}$ & $\begin{array}{l}\% \\
15.9 \\
31.8 \\
36.9\end{array}$ & $\begin{array}{c}\% \\
\text { None. } \\
\text { Do. } \\
\text { Do. }\end{array}$ \\
\hline
\end{tabular}

1 It was subsequently shown that some hydrogen sulfide was adsorbed on the walls of tube $K$ through which moist nitrogen was passed. 
TABLE 2.-Effect on wool of 266 hours' exposure in atmospheres of moist and dry air to the radiation of a glass-enclosed carbon arc

\begin{tabular}{|c|c|c|c|c|c|c|c|c|}
\hline Sample & $\begin{array}{c}\text { Total } \\
\text { nitrogen }\end{array}$ & $\begin{array}{l}\text { Ammo- } \\
\text { nia ni- } \\
\text { trogen }\end{array}$ & Cystine & $\begin{array}{l}\text { Total } \\
\text { sulfur }\end{array}$ & $\begin{array}{c}\text { Sulfur in } \\
\text { gases }\end{array}$ & $\begin{array}{c}\text { Total } \\
\text { sulfur ac } \\
\text { counted } \\
\text { for }\end{array}$ & $\begin{array}{l}\text { Alkali } \\
\text { solubil- } \\
\text { ity }\end{array}$ & $\begin{array}{l}\text { Sulfur as } \\
\text { sulfuric } \\
\text { acid (on } \\
\text { wool) }\end{array}$ \\
\hline Original sample... & $\begin{array}{c}\% \\
16.22\end{array}$ & $\underset{0.5}{\mathrm{mg} / \mathrm{g}}$ & $\begin{array}{l}\% \\
10.7\end{array}$ & $\begin{array}{l}\% \\
3.49\end{array}$ & $\%$ & $\begin{array}{c}\% \\
100.0\end{array}$ & $\begin{array}{l}\% \\
15.9\end{array}$ & $\%$ \\
\hline Exposed in dry air & 15. 87 & 1.7 & 5.3 & 3.16 & 0.06 & 194.5 & 74.5 & 0.41 \\
\hline Exposed in moist air & 15.81 & 1.4 & 5.5 & 3.00 & .05 & 189.5 & 75.7 & .39 \\
\hline
\end{tabular}

1 Tests showed that sulfuric acid was adsorbed on the walls of the tubes $K$ and $L$.

\section{DISCUSSION}

The available data can hardly be considered to be sufficient for a detailed explanation of the photochemical decomposition of wool. The results of the investigation, however, suggest several generalizations which appear to be useful in further elucidating the nature of some of these reactions.

It is evident from the curves in figure 3 that the evolution of hydrogen sulfide from wool which was irradiated in an atmosphere of nitrogen was greatly accelerated by the presence of moisture. It would appear at first sight, that a hydrolytic cleavage of the disulfide group of the cystine in wool occurred, similar to the one postulated by Crowder and Harris [6] to account for the degradation of wool in alkaline solutions. Hydrogen sulfide would then be formed according to the following reactions:

$$
\begin{array}{r}
-\mathrm{CH}_{2}-\mathrm{S}-\mathrm{S}-\mathrm{CH}_{2}-+\mathrm{H}_{2} \mathrm{O} \rightleftharpoons-\mathrm{CH}_{2} \mathrm{SH}+-\mathrm{CH}_{2} \mathrm{SOH} \\
-\mathrm{CHO}+\mathrm{H}_{2} \mathrm{~S}
\end{array}
$$

Assuming the above mechanism, it could be argued that in view of the great difficulty in obtaining absolutely dry wool, it is questionable whether all of the moisture was removed from the so-called dry sample at the start of these experiments. In other words, it would be difficult to tell whether the hydrogen sulfide obtained from the sample exposed in the atmosphere of dry nitrogen resulted from the decomposition of absolutely dry wool, or whether it was influenced by moisture which may still have been present.

If the photochemical decomposition of wool primarily involves a hydrolytic cleavage of the disulfide group, it must be pointed out, that of any moisture remaining on the fibers, some might be used up in photochemical reactions in the early stages of the exposure, but the rest would be rapidly removed by the warm, dry nitrogen which was continually passing over the sample. Under such conditions, there would be anticipated an initial, relatively rapid evolution of hydrogen sulfide, but the rate would soon decrease until practically no further evolution of gas occurred. That this is not so is indicated by the lower curve in figure 3.

If the decomposition proceeded according to equation 1 , it would also be expected that the decrease in cystine content would increase with increasing alkalinity of the sample since the sulfenic acid group (-SOH) becomes increasingly unstable with increasing $\mathrm{pH}$. That the reverse of this actually occurs was demonstrated by the authors in their earlier investigations [1] in which it was shown that the cystine content of a sample of wool treated with $0.1 \mathrm{Nhydrochloric}$ 
acid and irradiated 100 hours decreased 52 percent, whereas that of a sample treated with a $0.1 \mathrm{~N}$ solution of sodium carbonate and exposed for the same time decreased only 35 percent.

Finally, it is evident from the data in table 1 that although the moist sample lost more than four times as much sulfur as the dry sample, the cystine content of the former is only slightly lower than that of the latter. A large portion of the cystine is destroyed during the irradiation, and if it is assumed that the destruction involves a hydrolytic cleavage of the disulfide group, it is readily seen that an appreciable amount of water would also have had to be present in the dry sample.

It seems more likely that the action of the radiation on wool in an atmosphere of nitrogen primarily does not result in hydrolysis of the disulfide group. The data in table 1 show that the cystine contents, as determined by the highly specific Sullivan method [7], decreased about the same amount for the samples exposed in atmospheres of dry or of moist nitrogen, but the moist sample evolved four times as much hydrogen sulfide as the dry sample. This suggests that the primary action is probably an activation of the disulfide group, resulting in a change in the state of the sulfur and a corresponding lowering of the cystine content. A secondary process, involving the evolution of hydrogen sulfide from the irradiated wool then occurs.

The role of the water is of considerable interest. Although water does not appear to be essential to the process, which involves changing the state of the sulfur, it may play an important part in the secondary reaction. That it might conceivably catalyze the secondary reaction, or actually react with products of the primary reaction to liberate hydrogen sulfide, was demonstrated by immersing samples of irradiated wool in warm water. Considerable hydrogen sulfide was evolved, but under similar conditions, a significant amount of hydrogen sulfide was not obtained from untreated wool.

The photochemical reactions which occur when wool is irradiated in atmospheres of moist and dry air are even more complex. In addition to the attack on the sulfur, nitrogen groups are affected, as shown in table 2. The increase in ammonia nitrogen content results from photo-oxidation processes [8]. Only a relatively small amount of hydrogen sulfide is evolved, the bulk of it being immediately oxidized to sulfuric acid. The acid thus formed further catalyzes the photochemical decomposition [1], which accounts for the high alkali-solubilities. The moist sample lost more sulfur, but again the cystine contents and the alkali-solubilities of both the moist and dry sample were changed about the same amount.

\section{REFERENCES}

[1] J. Research NBS 1\%, 97 (1936) RP904; Am. Dyestuff Reptr. 25, 383 (1936).

[2] BS J. Research 4, 747 (1930) RP177.

[3] Physics 1, 352 (1931).

[4] J. Soc. Dyers Colourists 44, 14, 233 (1928).

[5] Compt. rend. 183, 596 (1926).

[6] J. Research NBS 16, 475 (1936) RP885; Am. Dyestuff Reptr. 25, 264 (1936).

[7] Public Health Rep. 86 (1930).

[8] J. Research NBS 13, 151 (1934) RP697; Am. Dyestuff Reptr. 23, 403 (1934).

[9] J. Am. Chem. Soc. 44, 2255 (1922).

Washington, October 15, 1937. 